JOURNAL OF BUSINESS

and entrepreneurial

studies

\title{
El estudio de pertinencia en la acreditación de las carreras administrativas en las instituciones de educación superior
}

\section{The study of relevance in the accreditation of administrative careers in higher education institutions}

Ing. Sofía Georgina Lovato Torres, Mgs

Universidad de Guayaquil

Guayaquil-Ecuador

sofia.lovatot@ug.edu.ec

ORCID https://orcid.org/0000-0001-5831-8554

Ing. María Teresa Mite Albán, Mgs

Universidad de Guayaquil

Guayaquil-Ecuador maria.mitea@ug.edu.ec

Universidad de Guayaquil

ORCID https://orcid.org/0000-0002-1607-5299

Recibido 10 octubre 2018 - Aceptado 4 diciembre - 2018 
El estudio de pertinencia en la acreditación de las carreras administrativas en las instituciones de educación superior

\section{Resumen}

La pertinencia de la oferta profesional en las instituciones de Educación Superior en Ecuador es un pilar fundamental para el desarrollo económico del país. La Universidad de Guayaquil ha cambiado en respuesta a la demanda laboral, entorno nacional, social y global, medir la gestión de calidad y realizar análisis de pertinencia que contribuye al desarrollo económico. El presente trabajo de investigación es de enfoque cualitativo y cuantitativo, se trata de analizar como el estudio de Pertinencia incide en la Acreditación de las Carreras Administrativas, Universidad de Guayaquil, este estudio es producto del trabajo en equipo realizado por el personal de Gestores Académicos de cada una de las carreras de la FCA, con el objetivo de obtener información que permita describir la Pertinencia de las carreras de la facultad relacionadas con las preferencias de los estudiantes de Bachillerato, la situación laboral de los graduados y egresados así como la demanda de los empleadores con respecto a los estudiantes, enmarcados en la zonas de planificación 5 y 8 del Plan Nacional del Buen Vivir (PNBV). Con este estudio se obtuvo información relevante de varios elementos que comprenden el estudio de Demanda actual aplicado a Bachilleres y el estudio de Empleabilidad debido a que los resultados del análisis están guiados a orientar las decisiones relacionadas al rediseño, aplicación y mejoramiento de la oferta académica de las Carreras
Administrativas, generando una sociedad del conocimiento emprendedora y líder, integrando los ejes sustantivos con una oferta profesional capaz de contribuir en los planes estratégico del Ecuador.

Palabras clave: Estudio de pertinencia, Acreditación, Ciencias Administrativas, y Educación Superior, Universidad de Guayaquil

\section{Abstract}

The relevance of the offer professional in the institutions of education Superior in Ecuador is a pilar fundamental for the development economic of the country. The University of Guayaquil has changed in response to the demand for labour, national, social and global, measure quality management and analysis of relevance that contributes to economic development. He present work of research is of approach qualitative and quantitative, is of analyze as the study of relevance affects in the accreditation of them racing administrative, University of Guayaquil, this study is product of the work in equipment made by the personal of managers academic of each an of them racing of the Faculty of Sciences administrative FCA; with the aim of obtaining information allow to describe the relevance of the Faculty careers related to the preferences of the students of secondary education, the employment situation of the graduates and alumni as well as the demand of employers with respect to students, in the areas of planning, 5 and 8 of the National Plan of the good living (PNBV). This study was 
obtained relevant information from the various elements that comprise the current demand applied to high school graduates and the study of employability due to the results of the analysis are guided to guide decisions related to the redesign, implementation and improvement of the academic offer of the administrative careers, creating a society of the knowledge entrepreneur and leader, integrating substantive shafts with a professional offer capable of contributing in the strategic plans of the Ecuador.

Keywords: study of relevance, accreditation, science administrative, and education Superior, University of Guayaquil

\section{Introducción}

Las Instituciones de Educación Superior se preocuparon en formar profesionales de acuerdo a la especialidad de los bachilleres $y$ en especial a las necesidades que percibían en el entorno social; esto propicio a que muchas facultades crearan Carreras sin realizar un adecuado estudio que permita sustentar los profesionales y el perfil que requerían las organizaciones; es así que en su momento, la Facultad de Ciencias Administrativas creó sus Carreras en un contexto muy diferente al actual, en la década del setenta en nuestro país no existían los órganos de regulación de la Educación Superior, por esa razón las carreras de Ingeniería Comercial y CPA de la FCA se crearon con requisitos vigentes expedidos por las autoridades que conformaban el Consejo Superior de la UG y con el devenir del tiempo la FCA creó carreras no tradicionales con base a la demanda del mercado empresarial.

Actualmente se debe realizar un análisis del entorno sus necesidades, y la demanda, analizando con base a encuesta a Bachilleres y la información proporcionada por el SNNA, por la Nivelación de la UG. y también a empleadores, graduados y egresados, con la finalidad de cumplir con los lineamientos establecidos para el criterio de Pertinencia, realizando estudios de demanda académica y profesional.

Dentro de los criterios que se deben cumplir en el Plan de Excelencia de la Universidad de Guayaquil, se tiene el de pertinencia de la institución, por lo tanto se considera importante el acercamiento pedagógico y pertinente en la producción de currículos contextualizados que se articulen con las demandas del entorno local, regional, y nacional, y cuya aplicación coadyuve a la organización, producción y gestión del conocimiento a través de una oferta académica actualizada, con procesos de vinculación y servicio a la sociedad, practicas preprofesionales de acuerdo al contexto de las carreras que fortalecen la formación profesional que se enmarquen dentro de las tendencias científico-tecnológicas desarrollando una internacionalización académica y participación en redes.

La educación superior debe responder a las expectativas y necesidades de la sociedad, a la planificación nacional, y al régimen de desarrollo, a la prospectiva de desarrollo científico, humanístico y tecnológico mundial, y a la diversidad cultural. Para ello, las instituciones de educación superior articularán su oferta 
El estudio de pertinencia en la acreditación de las carreras administrativas en las instituciones de educación superior

docente, de investigación y actividades de vinculación con la sociedad, a la demanda académica, a las necesidades de desarrollo local, regional y nacional, a la innovación y diversificación de profesiones y grados académicos, a las tendencias del mercado ocupacional local, regional y nacional, a las tendencias demográficas locales, provinciales y regionales; a la vinculación con la estructura productiva actual y potencial de la provincia y la región, y a las políticas nacionales de ciencia y tecnología. Por tal motivo, en cumplimiento con el art.107 de la LOES ,para mejorar su oferta académica y regularizar los diseños curriculares de cada una de las Carreras de la FCA , elabora el presente estudio donde se incluye un análisis sobre la demanda actual de los bachilleres en relación a la oferta académica de la Facultad, para lo cual se aplicó como herramienta una encuesta a los estudiantes de bachillerato de los 20 colegios más representativos determinados previamente en la preencuesta realizada a todos los estudiantes de las carreras.

Así como un análisis sobre la demanda laboral actual en relación a las carreras que ofrece esta Facultad, para lo cual se realizó una encuesta a 418 empresarios de la Zona 5 y Zona 8 con la finalidad de conocer su opinión acerca de las expectativas que tiene sobre los egresados de la Facultad de Ciencias Administrativas de la ciudad de Guayaquil y se concluyó con un estudio para Identificar las condiciones, experiencias laborales y la percepción de los egresados de esta Facultad, mediante una encuesta a 1036 Egresados de la Facultad de Ciencias Administrativas de la ciudad de Guayaquil. En consecuencia, los Estudios de Pertinencia constituyen un factor importante al momento de analizar los procesos educativos en la educación superior por su impacto no sólo en la instrucción sino también en los cambios que influyen en la economía, sociedad y cultura de la nación, permitiendo la formación de profesionales de alta calidad académica y humana que sean base para el cambio de la matriz productiva y coadyuven a la consecución de los más importantes objetivos del país General.

Analizar la pertinencia de las Carreras Administrativas para determinar su relación con las condiciones sociales, económicas, laborales y tecnológicas enmarcados en el PNBV y como incide en la Acreditación de las instituciones de educación superior, caso Universidad de Guayaquil.

Específicos.

- Diagnosticar los problemas, necesidades de los contextos y objetivos del Plan Nacional del Buen Vivir.

- Determinar la demanda actual de las Carreras relacionados con los bachilleres de los colegios seleccionados por criterio de la ciudad de Guayaquil.

- Identificar las condiciones y experiencias laborales de los egresados con relación a las competencias generales $y$ 
específicas desarrolladas en los procesos de formación académica en cada una de las Carreras.

- Identificar las demandas y requerimientos de los empleadores en relación a las competencias profesionales de los egresados y graduados de las diferentes carreras de la Facultad de Ciencias Administrativas de la Universidad de Guayaquil.

- Justificar la pertinencia de las Carreras de la FCA con base a los objetivos del PNBV.

La Pertinencia es un fenómeno promedio del cual se establecen las múltiples relaciones entre la universidad y el entorno de maneras diferentes, a través de diversas estructuras, tanto al interior de la misma como del entorno social. (Plata, 2003).

"La pertinencia de la educación superior, analizada se considera como función de su cometido y lugar en la sociedad, de sus funciones con relación a la enseñanza, la investigación y los servicios, así como de sus nexos con el mundo del trabajo, con el Estado y el financiamiento público, además de sus interacciones con otros niveles y formas de educación. La calidad es considerada como una noción pluridimensional, aunque se la puede definir como el ajustarse al logro de los objetivos que la institución ha fijado de antemano. Se aclara también que el concepto de calidad no se refiere exclusivamente a los productos, sino también a los procesos efectuados por el sistema, el cual funciona como un todo coherente para garantizar la pertinencia social". (Alcantara)

De acuerdo a la Organización de las Naciones Unidas para la Educación, Ciencia y Cultura la pertinencia de la Educación Superior debe evaluarse en función de la adecuación que la Sociedad espera, y los cambios que se generan al contribuir a través del conocimiento a las necesidades de la Sociedad. (UNESCO, 1998)

La Facultad de Ciencias Administrativas de la Universidad de Guayaquil cumpliendo los lineamientos del Plan de Excelencia busca fortalecer a la institución mediante la elaboración del presente estudio de Pertinencia desarrollado por los Gestores Académicos del Departamento de Acreditación.

Por tal motivo, en cumplimiento con el art.107 de la LOES, para mejorar su oferta académica y regularizar los diseños curriculares de cada una de las Carreras de la FCA Necesidades del entorno nacional.

1. ¿Cuáles son los problemas y necesidades de los contextos y objetivos del Plan Nacional del Buen Vivir -PNBV que abordará la profesión? 
El estudio de pertinencia en la acreditación de las carreras administrativas en las instituciones de educación superior

2. ¿Cómo están vinculadas las tecnologías de punta a los aprendizajes profesionales para garantizar la respuesta a los problemas que resolverá la profesión en los sectores estratégicos y de interés público?

\section{Figura 1.1. Matriz productiva}

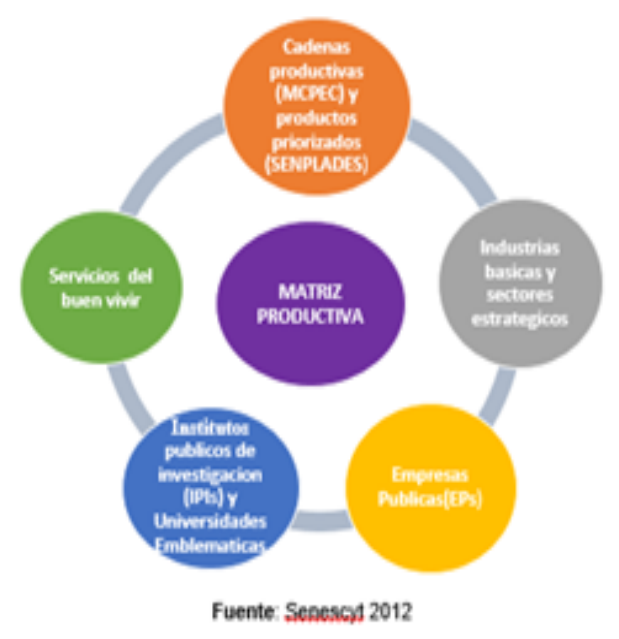

La Facultad de Ciencias Administrativas acorde a los requerimientos de la sociedad y en función de los planes de desarrollo nacional se involucra directamente en el establecimiento de una oferta académica coherente con los planes del estado ecuatoriano y de la sociedad a la que sirve.

La acreditación es una validación de evidencia quinquenal realizada por el CEAACES, para certificar la calidad de las instituciones de educación superior, de una carrera o programa educativo, sobre la base de una educación previa. (CEAACES)
En base al artículo mencionado, el propósito de la Comisión de Acreditación es:

Evaluar rigurosamente el cumplimiento de lineamientos, estándares y criterios de calidad a nivel nacional e internacional, a las carreras, programas, postgrados e instituciones, obligatorio e independiente, que definirá el CEAACES

El trámite para conseguir la acreditación tiene tres pasos: la evaluación interna (autoevaluación), que la hace la propia universidad y que incluye presentar un plan de mejoramiento; la evaluación externa, que comprende la revisión por parte del Consejo de Evaluación, Acreditación y Aseguramiento de la Calidad de la Educación Superior (CEAACES) y la acreditación, certificación que debe renovarse cada cinco años.

La calidad educativa supone el desarrollo de una cultura organizacional orientada hacia la evaluación y el mejoramiento continuo y la innovación, tanto del programa como de la institución, lo cual implica el despliegue de políticas, programas estratégicos, proyectos acciones y recursos que, integrados en planes de desarrollo, promuevan el cumplimiento de los enunciados misionales y de un ideal de excelencia con participación activa de la comunidad institucional. Por esto cobra mayor importancia que exista en cada institución una estructura, procesos $\mathrm{y}$ 
unos mecanismos que hagan seguimiento permanente a la pertinencia, eficiencia y eficacia del quehacer institucional y de los programas, en el marco de un proceso de autorregulación, cuya expresión visible ante la sociedad y el mundo académico es la acreditación temporal y su continua renovación. (Acreditación, 2013)

El requisito sine qua non para la acreditación de carreras, es la acreditación institucional de la universidad y su conjunto; es decir sólo pueden ser admitidas al proceso para la acreditación de carreras aquellas IES que hayan sido acreditadas institucionalmente. La acreditación de carreras es un proceso posterior a la acreditación institucional, que se realiza mediante convocatorias del CEAACES para evaluación de carreras con denominaciones similares. En cada convocatoria se establecerá un cronograma del proceso. Los siguientes pasos del proceso se realizarán de conformidad con el Reglamento de los Procesos de Evaluación y Acreditación expedido por el CEAACES y que figura en el portal institucional. (CEAACES, Modelo General para la Evaluación de Carreras con fines de Acreditación, 2011)

La Universidad de Guayaquil, la más poblada del país $(82.000$ alumnos matriculados), espera estar lista antes de octubre para recibir la visita de los evaluadores externos que certifiquen el trabajo realizado para lograr la acreditación y con ello ascender a la categoría $\mathrm{A}$, donde están ubicados los centros de estudios de excelencia.

En las 18 facultades y en las 83 carreras que tiene este centro de estudio superior hay comisiones que trabajan en la autoevaluación. Desde el vicerrectorado académico se coordina con ellas para trabajar en el proceso de acreditación.

\section{Materiales y métodos}

La presente investigación es de enfoque cualitativo y cuantitativo, descriptiva, exploratoria por que se realizó encuestas para el levantamiento de la información. Es de corte transversal debido a que la investigación fue desarrollada desde diciembre 2014 hasta el presente año.

Para estudiantes de Bachillerato de los colegios de la ciudad de Guayaquil, la población fue de 106.345 matriculados en el período 2014- 2015, de acuerdo a las cifras indicadas por la Subsecretaría de Educación de Guayaquil.

Para Empleadores, la población fue de 135.365 empresas en la Provincia de Guayas

Para egresados se consideró 6000 alumnos de los últimos 5 años.

El tamaño de la muestra fue:

Bachilleres: 18 colegios de los que mayormente provienen los estudiantes de la Facultad con un total de 5573 alumnos en la ciudad de Guayaquil

Empresas: se utilizó la técnica de muestreo aleatorio a 400 empresas, aunque se logró realizar a un grupo mayor que son 418 . 
El estudio de pertinencia en la acreditación de las carreras administrativas en las instituciones de educación superior

Egresados: se determinó una muestra de 1036 egresados.

\section{Resultados}

En el presente estudio se determinó que de la muestra de 5573 estudiantes de los 18 colegios de segundo de bachillerato, el $67,65 \%$ corresponde al género femenino mientras que el $32,35 \%$ corresponde al género masculino.

Cuando se requirió información con respecto a la preferencia de Instituciones de Educación Superior, se obtuvo los siguientes resultados previamente depurado con base a las preguntas anteriores: Las preferencias se dirigieron en un $52,07 \%$ a la Universidad de Guayaquil, siguiendo en preferencia la Escuela Superior Politécnica del Litoral con un $27,81 \%$ y en tercer lugar la Universidad Católica de Santiago de Guayaquil con el $9,83 \%$ de las preferencias, mientras que un $5,46 \%$ no contesto y un $4,83 \%$ indicó que lo haría en otras Instituciones. Estos porcentajes nos informan una preferencia importante por las IES públicas de más de la mitad de la muestra.

La mayor preferencia en relación al área del conocimiento, representada en el $27,08 \%$ de los encuestados, se presenta por carreras afines a la administración empresarial y sector económico, lo cual ratifica la tendencia que tienen los estudiantes de estos colegios para optar por una de las carreras de la Facultad de Ciencias Administrativas, en segundo lugar con un porcentaje de $16,59 \%$ las preferencias recayeron en el área de la Salud, y en un tercer lugar con un 14,43\% el área de la Informática.
La carrera de Ingeniería en Marketing y Negociación Comercial lidera con el $27,00 \%$ de los encuestados la tabla de preferencia de las carreras de Economía o Administración, seguida por las carreras de Ingeniería en Gestión empresarial y Contaduría Pública Autorizado (CPA), que tienen una preferencia del $14,32 \%$ y $13,62 \%$ de los encuestados, respectivamente. El 12,67 $\%$ muestra interés en la carrera de Ingeniería en Comercio Exterior, por encima de la tradicional carrera de Ingeniería Comercial con un 10,71\%. Las carreras de Tributación y Finanzas, Ingeniería Comercial y CPA A distancia virtual, en conjunto representan el 15\% de la preferencia.

Los resultados muestran que más de la mitad con un $50,73 \%$ de los encuestados consideran que la calidad de la educación en la Universidad de Guayaquil es buena, el 23,59\% manifiesta que es muy buena por lo que en un 70\% la imagen de la Universidad está bien posicionada en lo que respecta a su calidad.

Como resultado de la encuesta vemos que el área para el que mayormente las empresas han contratado recurso humano de la Facultad de Ciencias Administrativas de la Universidad de Guayaquil, es el área administrativa; seguida del área contable. Otras áreas para las cuales se ha considerado de manera importante el recurso humano son: área financiera, comercial o ventas, recursos humanos, compras. En menor nivel también existe presencia en las 
áreas mercadeo, transporte $\mathrm{y}$ distribución, producción, auditoría.

Como resultado de la encuesta se deduce que las habilidades que más valoran y requieren las empresas son: la habilidad para resolver problemas y la capacidad de aprender y actualizarse permanentemente, ambas en un $69 \%$. Seguido por la habilidad de buscar, procesar y analizar información de fuentes diversas, en un $66 \%$.

Así también la habilidad menos valorada o requerida por las empresas de acuerdo a la encuesta realizada es la responsabilidad social y compromiso ciudadano.

\section{Discusión}

Las necesidades del país están relacionadas con el cambio de la Matriz productiva contemplado en el Objetivo estratégico 10 del Plan Nacional del Buen Vivir, el estudio de las carreras concluye que la oferta de la FCA es pertinente a los cambios que plantea el Estado ecuatoriano, debido a que el objeto de estudio de las mismas tienen como denominador común un enfoque administrativo científico aplicable a todos los sectores estratégicos a desarrollar.

Las carreras de la FCA experimentaron en conjunto un incremento del $46 \%$ con respecto al periodo anterior, en todas las carrera la tendencia al alza se mantuvo debido a una asignación mayor de cupos por parte del SNNA.
Que, el 27.10\% de los encuestados eligió el área de estudio de la FCA de las cuales las más requeridas fueron Ingeniería en Marketing y Negociación comercial, Ingeniería en Gestión Empresarial, Contaduría Pública Autorizada e Ingeniería en Comercio Exterior; en contraste con la segunda fuente de información en las cuales se mantienen entre las cuatro más elegidas Contaduría Pública Autorizada, Ingeniería en Comercio Exterior e Ingeniería en Marketing y Negociación comercial y se introduce también Ingeniería Comercial siendo estas carreras las cuales tienen la mayor cantidad de matriculados de la FCA

\section{Conclusión}

Otro factor es la Demanda insatisfecha es que las Carreras que tienen el más alto porcentaje de insatisfacción por sobre el $80 \%$ son Ingeniería Comercial, Ingeniería en Marketing y Negociación comercial, y Contaduría Pública Autorizada. El mercado laboral indicaron que el 19\% de las empresas del país se encuentran en Guayas, del total nacional de empresas nueve de cada diez son micro y pequeñas empresas de las cuales el $14 \%$ se encuentran ubicadas en Guayaquil y las restantes medianas y grandes empresas en el resto del país. Las empresas de Guayaquil tienen un desarrollo en ventas del 11,90\% y según el estudio las áreas laborales que más solicitan personal de las carreras ofertadas son el área Contable, Comercial y Ventas, y Mercadeo pertenecientes a los siguientes sectores 
productivos como son Servicios, Comercio, Alimentos y Construcción que son los sectores con los porcentajes más altos de la muestra encuestada.

El grado de valoración de las competencias requeridas en las empresas por los empleadores fueron: Capacidad de comunicación oral y escrita; Habilidades para buscar, procesar y analizar información de fuentes diversas y Habilidad para resolución de problemas; en contraste con la valoración de estas mismas competencias adquiridas en la Universidad, es decir, que habilidades los empresarios piensan que son importantes desarrollarlas y asimilarlas en el proceso educativo tales competencias fueron: Habilidad de abstracción, análisis y síntesis, Capacidad de aprender y actualizarse permanentemente, Capacidad para la toma de decisiones.

\section{Referencias}

Acreditación, S. N. (2013).

LINEAMIENTOS PARA LA

ACREDITACIÓN DE PROGRAMAS

DE . Bogota- Colombia.

Alcantara, A. (s.f.). TENDENCIAS

MUNDIALES EN LA EDUCACION

SUPERIOR:EL PAPEL DE LOS

ORGANISMOS MULTILATERALES.

http://www.ses.unam.mx/integrantes/alc

antara/publicaciones/Tendencias.pdf.
CEAACES. (2011). Modelo General para la Evaluación de Carreras con fines de Acreditación.

CEAACES. (s.f.). Art. 95.

Plata, L. (2003). La pertinencia de la educación superior. Elementos para su comprensión. Revista de la Educación Superior, 120.

UNESCO. (1998). DECLARACION MUNDIAL SOBRE LA EDUCACION SUPERIOR.

PERIOR:EL PAPEL DE LOS

ORGANISMOS MULTILATERALES. http://www.ses.unam.mx/integrantes/alc antara/publicaciones/Tendencias.pdf. 